\title{
A Modified Control Method for Congested Traffic in Car-following Model
}

\author{
Yu Cui \\ Faculty of Science \\ Ningbo University \\ Ningbo, China \\ E-mail: cui163163163@163.com
}

\author{
Hong-xia Ge \\ Faculty of Maritime and Transportation \\ Ningbo University \\ Ningbo, China \\ E-mail: gehongxia@nbu.edu.cn
}

\begin{abstract}
In order to describe the car-following behavior more actually in real traffic, an extended car-following model incorporating the headway of arbitrary number of vehicles that proceed and the relative velocity is proposed from the viewpoint of control. The stability condition of the extended model is obtained by using the linear stability theory. The modified control signals will play an effect only if the traffic is in congested state. The results of simulation are accordance with our theoretical analysis.
\end{abstract}

Keywords- car-following model; control method; arbitrary number; stability analysis

\section{INTRODUCTION}

Suppression of the traffic congestion has attracted much attention [1-2]. An increasing number of traffic models have been developed and empirically tested. In 1995, Bando et al.[3] proposed an optimal velocity (OV) model for characterizing the car-following behavior. We recognize the OV model as a basic model for studying the phenomena of the traffic flow. In 1999, Konishi et al. [4] proposed a coupled-map (CM) car-following model (KKH model), which introduced the version of the decentralized delayedfeedback control scheme for suppressing the traffic jam. What is worth pointing out is that Zhao and Gao [6] presented another simple strategy to suppress the congested state in the traffic system with a control signal, which incorporates the effect of velocity difference between the preceding and the considered vehicle. Some research related to the control signal is carried out [4,5].

In order to improve the model with the control signal taking into account the two velocity differences, we investigate the property of extended optimal velocity model in which a driver considers arbitrary number of vehicles that proceed with extended control method.

\section{MODEL}

In the paper, we extended the OV model as following

$$
\left\{\begin{array}{c}
\frac{d v_{i}(t)}{d t}=a\left\{V^{o p}\left(y_{i+1}(t), y_{i+2}(t), \cdots, y_{i+n}(t)\right)-v_{i}(t)\right\} \\
\frac{d y_{i+1}(t)}{d t}=v_{i+1}(t)-v_{i}(t) \\
\frac{d y_{i+2}(t)}{d t}=v_{i+2}(t)-v_{i+1}(t) \\
\vdots \\
\frac{d y_{i+n}(t)}{d t}=v_{i+n}(t)-v_{i+n-1}(t)
\end{array}\right.
$$

Where $a>0$ is the sensitivity of a driver with response to the difference between the optimal and current velocities, $v_{i}(t)$ is velocity of the $i$ th vehicle at time $t$ and

$$
y_{i+n}(t)=x_{i+n}(t)-x_{i+n-1}(t)
$$

for $n=1,2,3, \cdots, N$ are headway of the $(i+n)$ th vehicle. Vehicles are numbered such that the $(i+2)$ th vehicle precedes the second vehicle. The model with $n=2$ is that proposed by Ge [12]. The extended $\mathrm{OV}$ function is $V^{o p}\left(y_{i+1}(t), y_{i+2}(t), \cdots, y_{i+n}(t)\right)$ represents an optimal velocity of the $i$ th vehicle and is described as

$$
V^{o p}(\bar{y}(t))=\tanh \left(\bar{y}(t)-h^{d}\right)+\tanh \left(h^{d}\right)
$$

Where $\bar{y}=\sum_{k=1}^{n} a_{k} y_{k}(t)$ which is named as the weighted headway and $a_{k}$ is the weighted coefficients of $y_{k}(t)$. We assume that $a_{k}$ have the following properties:

(1) $a_{k}(k=1,2,3, \cdots, N)$ decrease monotonically with increasing $k$ and $a_{k}>a_{k+1}$. As the distance between the car ahead and the considered car increases, the effect of cars ahead on the car motion reduces gradually.

(2) $\sum_{k=1}^{n} a_{k}=1$ Here we select $a_{k}=6 / 7^{k}(k \neq n)$ and $a_{n}=1 / 7^{n-1}$ for $k=n$.

We suppose the desired velocity of vehicles and comprehensive distance is $v^{*}$ and $y^{*}$, so the steady state of the system can be expressed as 


$$
(v, y)=\left(v^{*}, y^{*}\right)^{T} .
$$

\section{LINEAR STABILITY ANALYSIS}

We apply the linear stability theory to analyze the extended model which is described by above Eq. Let model Eq. be linearized around steady state and the linearized vehicular dynamics can be rewritten as follows:

$$
\left\{\begin{array}{c}
\frac{d v_{i}^{0}(t)}{d t}=a\left(\Lambda_{1} y_{i+1}^{0}(t)+\Lambda_{2} y_{i+2}^{0}(t)+\cdots+\Lambda_{n} y_{i+n-1}^{0}(t)-v_{i}^{0}(t)\right) \\
\frac{d y_{i+1}^{0}(t)}{d t}=v_{i+1}^{0}(t)-v_{i}^{0}(t) \\
\frac{d y_{i+2}^{0}(t)}{d t}=v_{i+2}^{0}(t)-v_{i+1}^{0}(t) \\
\vdots \\
\frac{d y_{i+n}^{0}(t)}{d t}=v_{i+n}^{0}(t)-v_{i+n-1}^{0}(t)
\end{array}\right.
$$

where

$$
\begin{gathered}
\Lambda_{1}=\left.\frac{\partial V\left(y_{i+1}(t), y_{i+2}(t), \cdots, y_{i+n}(t)\right)}{\partial y_{i+n}(t)}\right|_{y_{n+1}(t)=y^{*}, \cdots, y_{i+n}=y^{*}}, \\
\Lambda_{2}=\left.\frac{\partial V\left(y_{i+1}(t), y_{i+2}(t), \cdots, y_{i+n}(t)\right)}{\partial y_{i+n-1}(t)}\right|_{y_{n+1}(t)=y^{*}, \cdots, y_{i+n}=y^{*}}, \\
\cdots, \Lambda_{n-1}=\left.\frac{\partial V\left(y_{i+1}(t), y_{i+2}(t), \cdots, y_{i+n}(t)\right)}{\partial y_{i+2}(t)}\right|_{y_{n+1}(t)=y^{*}, \cdots, y_{i+n}=y^{*}} \\
\Lambda_{n}=\left.\frac{\partial V\left(y_{i+1}(t), y_{i+2}(t), \cdots, y_{i+n}(t)\right)}{\partial y_{i+1}(t)}\right|_{y_{n+1}(t)=y^{*}, \cdots, y_{i+n}=y^{*}} . \\
v_{i}^{0}(t)=v_{i}(t)-v^{*}, v_{i+1}^{0}(t)=v_{i+1}(t)-v^{*}, \cdots, \\
v_{i+n}^{0}(t)=v_{i+n}(t)-v^{*} \text { and } y_{i+1}^{0}(t)=y_{i+1}(t)-y^{*}, \\
y_{i+2}^{0}(t)=y_{i+2}(t)-y^{*}, \cdots, y_{i+n}^{0}(t)=y_{i+n}(t)-y^{*} .
\end{gathered}
$$

According to the control theory, the vehicular dynamics described by above Eq can be rewritten as a linear timeinvariant system as follows:
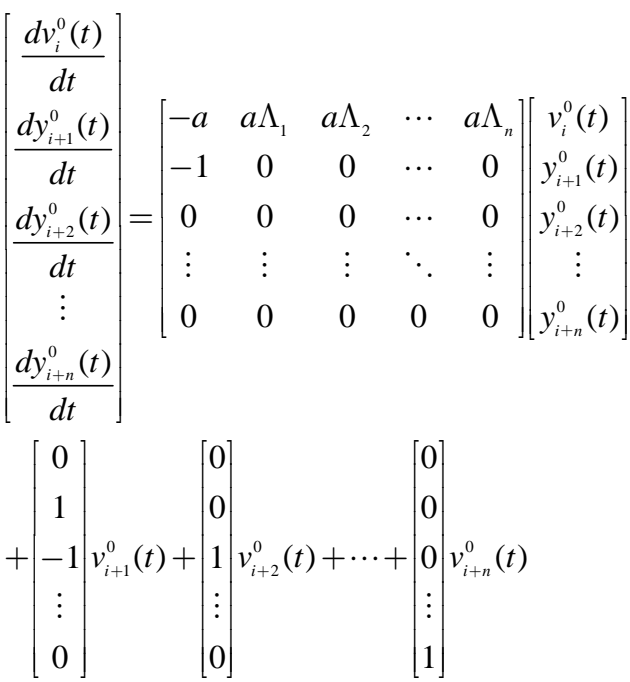

Taking Laplace transformation as L, we have

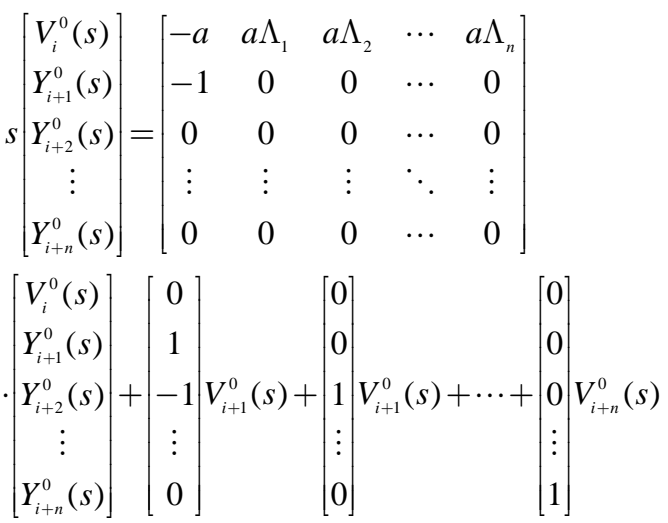

Where $V_{i+n}^{0}(s)=L\left(v_{i+n}^{0}(t)\right), Y_{i+n}^{0}(s)=L\left(y_{i+n}^{0}(t)\right)$.

From the above Eq we can obtain the transfer relationship

$$
\begin{gathered}
V_{i}^{0}(s)=\frac{a\left(\Lambda_{1}-\Lambda_{2}\right)}{s^{2}+a s+a \Lambda_{1}} V_{i+1}^{o}(s)+\frac{a\left(\Lambda_{2}-\Lambda_{3}\right)}{s^{2}+a s+a \Lambda_{1}} \\
\cdot V_{i+2}^{o}(s)+\cdots+\frac{a \Lambda_{n}}{s^{2}+a s+a \Lambda_{1}} V_{i+n}^{o}(s)+\frac{a}{s^{2}+a s+a \Lambda_{1}} \\
\cdot\left[\Lambda_{1} Y_{i+1}^{0}(s)+\Lambda_{2} Y_{i+2}^{0}(s)+\cdots+\Lambda_{n-1} Y_{i+n-1}^{0}(s)\right] \\
V_{i}^{0}(s)=T(s) \cdot V_{i+1}^{0}(s)
\end{gathered}
$$

Where transfer function is $\frac{a\left(\Lambda_{1}-\Lambda_{n}\right)}{s^{2}+a s+a \Lambda_{1}}$ and the characteristic polynomial is $d(s)=s^{2}+a s+a \Lambda_{1}$.

In order to keep from the traffic congestion, we should keep $d(s)$ stable. The $H_{\infty}$ norm of transfer function $T(s)$ is equal to or less than 1 . Through derivation we can obtain the condition

$$
a \geq 2 \Lambda_{1}, \quad \Lambda_{1}>\Lambda_{n} .
$$

According to the model proposed by Ge [18], traffic jams will never occur. 


\section{STABILITY ANALYSIS WITH CONTROL SignALS}

In order to effectively alleviate the traffic jams, a control signal term, $u_{i}(t)$, is added to vehicular dynamics described as (1) by model Eq. as follows:

$$
\left\{\begin{array}{c}
\frac{d v_{i}(t)}{d t}=a\left\{V^{o p}\left(y_{i+1}(t), y_{i+2}(t), \cdots, y_{i+n}(t)\right)\right. \\
\left.-v_{i}(t)\right\}+u_{i}(t) \\
\frac{d y_{i+1}(t)}{d t}=v_{i+1}(t)-v_{i}(t) \\
\frac{d y_{i+2}(t)}{d t}=v_{i+3}(t)-v_{i+2}(t) \\
\vdots \\
\frac{d y_{i+n}(t)}{d t}=v_{i+n}(t)-v_{i+n-1}(t)
\end{array}\right.
$$

The extended control signals $u_{i}(t)$ is

$u_{i}(t)=\sum_{k=1}^{n} a_{k}\left(v_{i+k}(t)-v_{i+k-1}(t)\right)$

Where $u_{i}(t)$ considers the headway of arbitrary number of successive vehicles in front, $a_{k}$ is the feedback gains and same to weighted coefficient of $y_{k}(t)$. Around the steady state referred to model Eq., vehicle dynamics described by Eq.(1) with control signals defined by above Eq. can be written as

$$
\left\{\begin{array}{c}
\frac{d v_{i}^{0}(t)}{d t}=a\left\{V^{o p}\left(y_{i+1}^{0}(t), y_{i+2}^{0}(t), \cdots, y_{i+n-1}^{0}(t)\right)\right. \\
\left.-v_{i}^{0}(t)\right\}+u_{i}^{0}(t) \\
\frac{d y_{i+1}^{0}(t)}{d t}=v_{i+1}^{0}(t)-v_{i}^{0}(t) \\
\frac{d y_{i+2}^{0}(t)}{d t}=v_{i+2}^{0}(t)-v_{i+1}^{0}(t) \\
\vdots \\
\frac{d y_{i+n}^{0}(t)}{d t}=v_{i+n}^{0}(t)-v_{i+n-1}^{0}(t)
\end{array}\right.
$$

$u_{i}(t)$ can be changed to $u_{i}(t)=\sum_{k=1}^{n} a_{k} \frac{d y_{i+k}^{0}(t)}{d t}$.

We have the following transfer relationship as the same way to deal with the above equations:

$$
\begin{aligned}
V_{i}^{0}(s)= & \frac{a\left(\Lambda_{1}-\Lambda_{2}\right)+\left(k_{1}-k_{2}\right) s}{s^{2}+\left(a+k_{1}\right) s+a \Lambda_{1}} V_{i+1}^{0}(s)+ \\
& \frac{a\left(\Lambda_{2}-\Lambda_{3}\right)+\left(k_{2}-k_{3}\right) s}{s^{2}+\left(a+k_{1}\right) s+a \Lambda_{1}} V_{i+2}^{0}(s)+
\end{aligned}
$$

$$
\begin{aligned}
& \frac{a\left(\Lambda_{3}-\Lambda_{4}\right)+\left(k_{3}-k_{4}\right) s}{s^{2}+\left(a+k_{1}\right) s+a \Lambda_{1}} V_{i+3}^{0}(s)+\cdots \\
& +\frac{a\left(\Lambda_{n-1}-\Lambda_{n}\right)+\left(k_{n-1}-k_{n}\right) s}{s^{2}+\left(a+k_{1}\right) s+a \Lambda_{1}} V_{i+n-1}^{0}(s)+ \\
& \frac{a \Lambda_{n}+k_{n} s}{s^{2}+\left(a+k_{1}\right) s+a \Lambda_{1}} V_{i+n}^{0}(s)+a \sum_{k=1}^{n} Y_{i+k}^{0}(0) \Lambda_{k} \\
& V_{i}^{0}(s)=T^{*}(s) \cdot V_{i+1}^{0}(s)
\end{aligned}
$$

Where transfer function $T^{*}(s)$ is $\frac{a\left(\Lambda_{1}-\Lambda_{n}\right)+\left(k_{1}-k_{n}\right) s}{s^{2}+\left(a+k_{1}\right) s+a \Lambda_{1}}$, the characteristic polynomial $d^{*}=s^{2}+\left(a+k_{1}\right) s+a \Lambda_{1}$. In order to keep $d^{*}(s)$ be stable, we should confirm that $a+k_{1}>0$ and $\Lambda_{n}>0$ in the view of Hurwitz stability criterion. Because of the OV function characteristics of monotonously increase (i.e. $\Lambda_{n}>0$ ) and $a>0$, the condition for $d^{*}(s)$ to be stable is $k_{1}>-a$, then consider

$$
\left\|\mathrm{T}^{*}(\mathrm{~s})\right\|_{\infty} \leq 1
$$

and $\left\|\mathrm{T}^{*}(\mathrm{~s})\right\|_{\infty}=\sup _{\omega \in[0, \infty)} \mathrm{T}^{*}(\mathrm{~s}) \nless 1$.We make transform as follows:

$$
\begin{aligned}
& \sqrt{T^{*}(j w) \cdot T^{*}(-j w)}=\sqrt{\frac{\left(a\left(\Lambda_{1}-\Lambda_{n}\right)\right)^{2}+\left(\left(k_{1}-k_{n}\right) w\right)^{2}}{\left(a \Lambda_{n}-w^{2}\right)^{2}+\left(\left(a+k_{1}\right) w\right)^{2}}} \leq 1, . \\
& \text { Let } f(w)=\sqrt{\frac{a^{2}\left(\Lambda_{1}-\Lambda_{n}\right)^{2}+\left(\left(k_{1}-k_{n}\right) w\right)^{2}}{\left(a \Lambda_{n}-w^{2}\right)^{2}+\left(\left(a+k_{1}\right) w\right)^{2}}} .
\end{aligned}
$$

Obviously, we can easily get $f(0)<1$.

Then we should keep $f(w) \leq 1$ for every $w \in(0, \infty)$, we only need make $d^{*}(s)>0$. We can get the sufficient condition $k_{1} \geq \Lambda_{1}-a / 2, k_{1}>-a, k_{1}>k_{n}$

Thus, we can summarize through control signals stability analysis as follows:

Theorem 1. The feedback control signals considered the $\mathrm{N}$-front vehicles can suppress the traffic jams effectively under the traffic disturbance in the single lane road and the conditions are $k_{1} \geq \Lambda_{1}-a / 2, k_{1}>-a, k_{1}>k_{n}$.

\section{NUMERICAL SIMULATIONS}

In the two velocity difference model [17], it was shown that the traffic congestion is decreased due to adding two velocity difference effects into the model. In our model, considering $\mathrm{N}$-front velocity difference effect, the result is better than previous one. In this paper the boundary condition is the periodic condition and the parameters are set as $y^{*}=7.02 m, v^{*}=20 m / s, a=2 s^{-1}$. It is assumed that all the vehicles have the same parameters. The 
initial positions and speed which are set to be $x_{i}(0)=\sum_{j=i+1}^{N} y_{j}(0), v_{i}(0)=v_{i}^{*}, i=1,2, \cdots, N$. The vehicles run without control signals for $0 \leq i \leq 2000$, and assuming the lead vehicle suddenly stops for $2 \leq i \leq 52$.

It is shown that Fig. 1 (a) is spatiotemporal pattern of the traffic flow in an uncontrolled system and the horizontal axis defined by $x_{i}=x_{1}-x_{i}$, which represents a distance between the first vehicle and the following one. The vertical axis notes the time. It is observed that the traffic congestion occurs in Fig. 1. The region of traffic jams decreases with time. In the following research, we will give the traffic system with the extended control method signals.

It is noted that there is only smaller oscillating by our method, compared with the new non-control system. These simulations show that the extended car-following model with the control method by considering the headway of arbitrary number of vehicles that proceed can be used to suppress the traffic congestion and the traffic system exhibits better behavior.

\section{CONCLUSIONS}

This paper proposes an extended control method for suppression the traffic jam based on the pioneer work of $\mathrm{Ge}$ [6]. The effect of our control signal on traffic congestion is investigated. We have obtained the stability condition for our model with the corresponding control signal. The simulation shows that the car-following model considering $\mathrm{N}$-front vehicles has a better effect than previous ones.

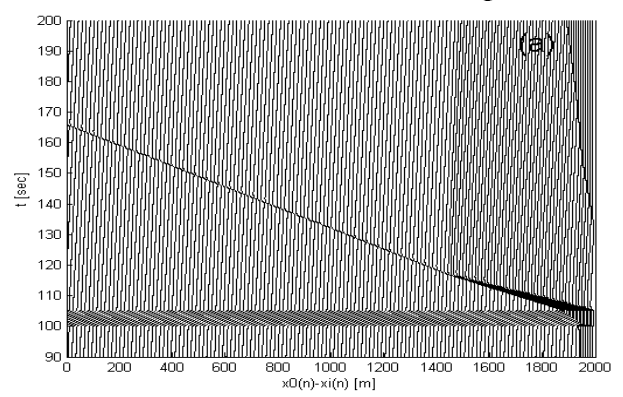

Additionally, the detailed analysis of parameters and the proper number of vehicles preceded are also our future work.

\section{ACKNOWLEDGMENT}

Project supported by the National Natural Science Foundation of China (Grant Nos.11372166, and 61074142), the Scientific Research Fund of Zhejiang Provincial, China (Grant No.LY13A010005), Disciplinary Project of Ningbo, China (Grant No.SZXL1067) and the K.C. Wong Magna Fund in Ningbo University, China. Government of the Hong Kong Administrative Region, China No.119011.

\section{REFERENCES}

[1] T.Q. Tang, C.Y. Li, H.J. Huang and H.Y. Shang (2012)” A new fundamental diagram theory with the individual difference of the driver's perception ability”. Nonlinear Dyn. 67, 2255-2265.

[2] Y.F. Li, D.H. Sun, W.N. Liu, M. Zhang, M. Zhao, X. Y. Liao and L. Tang(2011): Erratum to"Modeling and simulation for microscopic traffic flow based on multiple headway, velocity and acceleration difference”. Nonlinear Dyn. 66, 15-28.

[3] M. Bando, K. Hasebe, A. Nakayama, A. Shibata, Y. Sugiyama(1995)"Dynamical model of traffic congestion and numerical simulation”. Phys.Rev. E 51, 1035-1042.

[4] K. Konishi, H Kokame, K. Hirata(1999)"Coupled map car-following model and its delayed-feedback control”. Phys.Rev.E, 60 4000-4007.

[5] X.M Zhao and Z.Y. Gao(2006)"A control method for congested traffic induced by bottlenecks in the coupled map car-following model” Physica A 366, 513-522..

[6] H.X. Ge, S.Q. Dai, L.Y. Dong and Y. Xue(2004)”Stabilization effect of traffic flow in an extended car-following model based on an intelligent transportation system application”Phys.Rev.E. 70, 066134066140

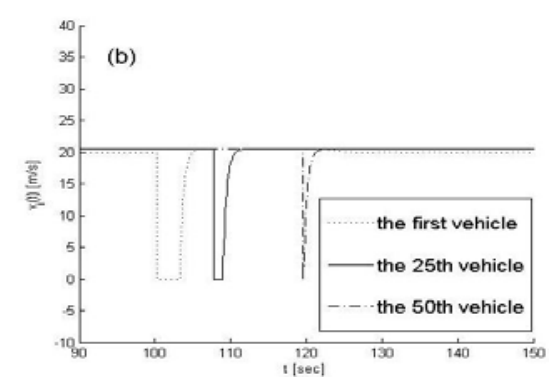

Fig1. Spatiotemporal pattern of traffic system after $t=90 \mathrm{~s}$ : (a) is the uncontrolled system. (b) is the temporal velocity of the first, $25^{\text {th }}$ and $50^{\text {th }}$ vehicle corresponding to (a).
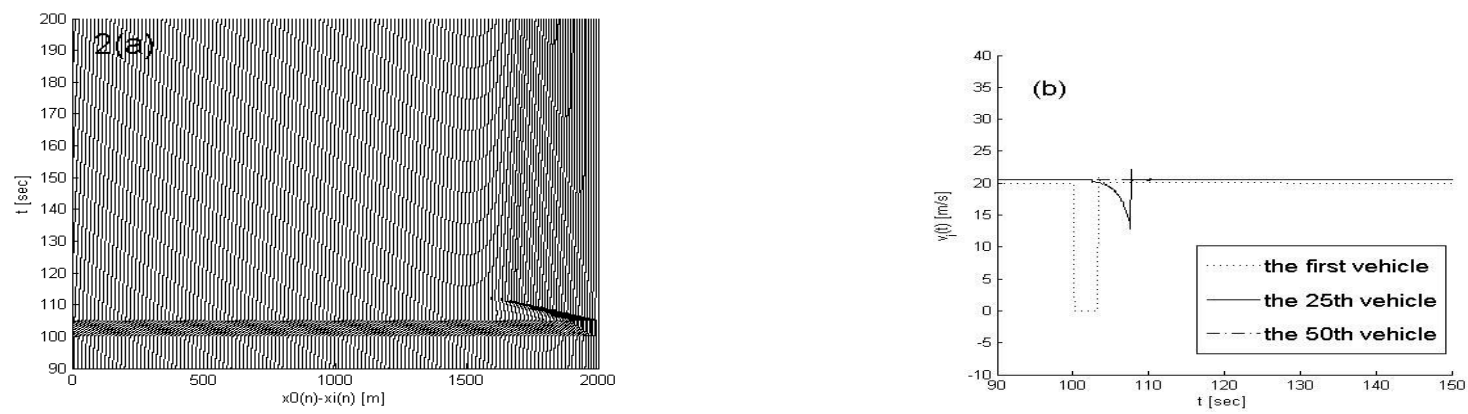

Fig.2. Spatiotemporal pattern of traffic system after $t=90 \mathrm{~s}$ : (a) is the controlled system. (b) is the temporal velocity of the first, $25^{\text {th }}$, and50 $0^{\text {th }}$ vehicle corresponding to (a) 